\title{
Intradural extramedullary capillary hemangioma: A case report and review of the literature
}

\author{
KEJUN ZHU and DENGWEI HE \\ Department of Spinal Surgery, Lishui Center Hospital, Zhejiang University, Lishui, Zhejiang 323000, P.R. China \\ Received January 26, 2015; Accepted February 16, 2016
}

DOI: $10.3892 / 01.2016 .4320$

\begin{abstract}
Capillary hemangiomas are benign tumors frequently located in the skin and other soft tissues during childhood. However, intradural extramedullary capillary hemangioma is extremely rare. The current study reports a rare case of intradural extramedullary capillary in a 59-year-old woman who presented with backache and right lower limb numbness that had progressed over 20 days. Magnetic resonance imaging of the thoracic spine showed an ill-defined mass at the T8 level. All other examinations were normal. The patient underwent surgery and recovered successfully. Histopathological examinations showed that the tumor exhibited the typical histological findings of capillary hemangioma, as it was comprised of a proliferation of capillary-sized vessels. The patient remains alive with no evidence of tumor metastasis and recurrence at 24 months post-surgery.
\end{abstract}

\section{Introduction}

The majority of capillary hemangiomas occur superficially in the cutaneous and mucosal tissues of the face, neck, nasal vault and oral cavity, particularly in children $(1,2)$. Within the nervous system, these tumors have been found in the spinal cord, the cauda equina and the nerve roots (3-5). According to the statistics and data analyses performed in a previous study, vascular lesions comprise $6-7 \%$ of all spinal intradural tumors, including capillary hemangioma, cavernous angiomas, and arteriovenous and venous malformations (6). Furthermore, capillary hemangiomas that occur in the intradural extramedullary space are extremely rare. Due to the rarity of such tumors, incidence and mortality rates remain unclear. Intradural capillary hemangiomas commonly behave as space-occupying tumors, which may subsequently lead to chronic progressive radiculopathy or myelopathy (7). Imaging

Correspondence to: Mr. Dengwei He, Department of Spinal Surgery, Lishui Center Hospital, Zhejiang University, 289 Kuocang Road, Lishui, Zhejiang 323000, P.R. China

E-mail: hedengwei9@126.com

Key words: intradural, extramedullary, capillary hemangioma, thoracic examination, particularly magnetic resonance imaging (MRI), aids in the determination of the size and location of the mass, and whether the mass is benign or malignant (7). Complete resection is considered as the most effective treatment of intradural capillary hemangioma (6). The current study presents a rare case of intradural extramedullary capillary hemangioma in a 59-year-old woman, and reviews the previously reported cases in the literature.

\section{Case report}

Case presentation. A 59-year-old woman presented to Lishui Center Hospital due to backache and right lower limb numbness that had progressed over 20 days. The patient had no history of coronary disease, obesity, type II diabetes mellitus or hypertension. Written informed consent was obtained from the patient for the publication of the present study.

Clinical analysis and surgery. Upon physical examination, superficial sensations of the right lateral crural region and dorsum pedis were decreased. Knee and ankle reflexes were increased. The straight leg raise test was performed to $90^{\circ}$ in each lower extremity. Thoracic MRI (Magnetom Symphony; Siemens, Munich, Germany) revealed an ovoid, intradural, extramedullary mass, $2 \mathrm{~cm}$ in length and $1 \mathrm{~cm}$ in diameter, at the T8 level. The tumor was isointense with the spinal cord on T1-weighted images and slightly hyperintense on T2-weighted images (Fig. 1A and B). The tumor showed markedly homogeneous enhancement on contrast-enhanced T1-weighted images (Fig. 2). A pre-operative diagnosis of meningioma was considered due to the MRI features. Further examinations, including routine blood [hemoglobin, $12 \mathrm{~g} / 1$ (normal range, 11-16 g/l); red blood cell count, $3.63 \times 10^{12} / 1$ (normal range, $3.5-5.5 \times 10^{12} / 1$ ); white blood cell count, 4,000 cells $/ \mathrm{ml}$ (normal range, $4,000-10,000$ cells $/ \mathrm{ml}$ ); platelet count, 1,780 cells/ml (normal range, 1,000-3,000 cells $/ \mathrm{ml}$ ); glucose level, $5.75 \mathrm{mmol} / 1$ (normal range, 3.9-6.1 mmol/1)], coagulation function [prothrombin time, $13.4 \mathrm{sec}$ (normal range, 10.5-14.0 sec); activated partial thromboplastin time, $30.5 \mathrm{sec}$ (normal range, 23.5-36.0 sec); thrombin time, $15.8 \mathrm{sec}(14.0-21 \mathrm{sec})$; international normalized ratio, 0.9 (normal range, $0.8-1.2)]$, serum electrolyte $\left[\mathrm{Na}^{+}, 142.6 \mathrm{mmol} / 1\right.$ (normal range, 137-147 mmol/1); $\mathrm{K}^{+}, 4.06 \mathrm{mmol} / 1$ (normal range, $3.5-5.3 \mathrm{mmol} / \mathrm{l}$ ); $\mathrm{Mg}^{2+}, 0.92 \mathrm{mmol} / 1$ (normal range, 0.64-1.25 mmol/l); $\mathrm{Cl}^{-}, 108 \mathrm{mmol} / \mathrm{l}$ (normal 


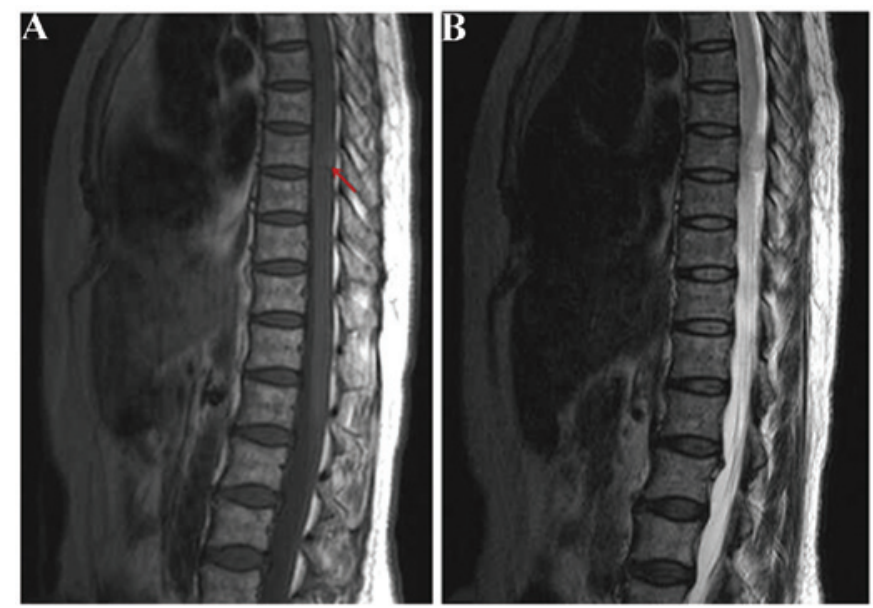

Figure 1. On thoracic magnetic resonance imaging, the tumor (arrow) was (A) isointense with the spinal cord on T1-weighted images and (B) slightly hyperintense on T2-weighted images.

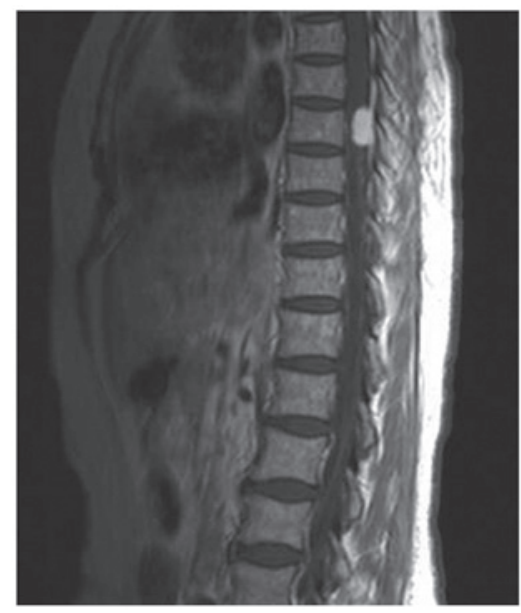

Figure 2. Contrast-enhanced T1-weighted thoracic magnetic resonance imaging showing a markedly homogeneous enhancing intradural extramedullary mass at the L8 level.

range, 99-110 $\mathrm{mmol} / \mathrm{l}$ ); $\mathrm{Ca}^{2+}, 2.04 \mathrm{mmol} / 1$ (normal range, 2.03-2.67 $\mathrm{mmol} / \mathrm{l})$ ], electrocardiogram [heart rate, 68 beats per min (normal range, 60-100 beats per min)] and lung function tests [forced vital capacity (FVC), 2,680 ml (normal range, $>2400 \mathrm{ml}$ ); forced expiratory volume in $1 \mathrm{sec}$ (FEV1), 2,270 $\mathrm{ml}$ (normal range, >2,000ml); FEV1/FVC ratio, $84 \%$ (normal range, $>83 \%$ ); peak expiratory flow rate, $5.92 \mathrm{l} / \mathrm{sec}$ (normla range, $5.5 \mathrm{l} / \mathrm{sec}$ )], were all within normal limits. The patient underwent a T7-T8 laminectomy. Upon completion of the exposure of the dural sac, a $1 \times 1.5 \times 2-\mathrm{cm}^{3}$ ovoid, well-circumscribed mass was identified, densely attached to the inner surface of the dura matter. The tumor was completely resected. Resected tissue speciments were formalin-fixed, paraffin-embedded and cut into $4-\mu \mathrm{m}$ sections. Histopathological examinations using hematoxylin and eosin staining (Sinopharm Chemical Reagent Co., Ltd., Shanghai, China) revealed that the tumor exhibited the typical histological findings of a capillary hemangioma, as it was comprised of a proliferation of capillary-sized vessels (Fig. 3).

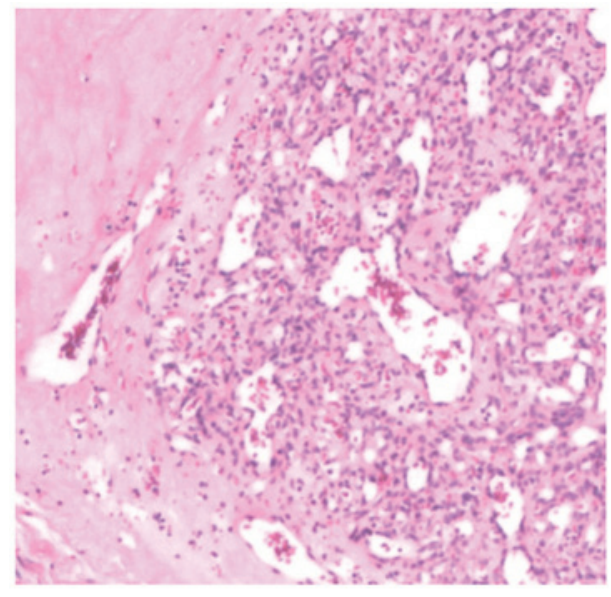

Figure 3. Hematoxylin and eosin staining of a tumor consisting of a myriad of tightly packed capillary-sized vessels lined by endothelial cells (magnification, $\mathrm{x} 100)$.

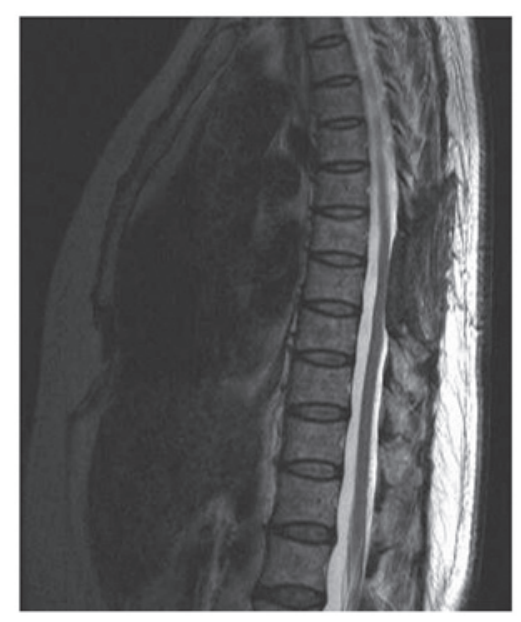

Figure 4. Thoracic magnetic resonance imaging of the patient showing no recurrence after 2 years of follow-up.

Follow-up. The patient was discharged on the 14th post-operative day following an uneventful recovery. After the surgery, the patient experienced significant relief from the backache. After 2 months, sensation and reflexes of the knee and ankle were normal. After 2 years of post-operative follow-up, no evidence of a recurrent tumor was evident on T2-weighted images (Fig. 4). The patient is currently managed with close clinical and radiological follow-up.

\section{Discussion}

Capillary hemangioma is a common, benign soft-tissue tumor that occurs in the skin or mucosa of the head and neck, and is occasionally located at the intradural extramedullary space (6). To the best of our knowledge, $>20$ cases of intradural extramedullary capillary hemangioma have been reported $(2,7)$. Based on findings from a previous study, the blood vessels of spinal intradural capillary hemangioma may arise from the inner surface of the dura, the pial surface of the spinal cord or the nerve roots (7). In the present case, it was found that the inner surface of the dura was the site of origin. When the 
tumor is small, patients often present with no clear clinical symptoms. With the gradual growth of the tumor, patients present with different symptoms, such as pain, numbness of limb, lower limbs hypesthesia and other neurological symptoms, according to the tumor size and location (7).

Imaging examinations, particularly MRI, are useful and effective tools in the evaluation of intradural extramedullary tumors. According to the study by Shin et al, the MRI findings of spinal capillary hemangiomas show isointensity with the spinal cord on T1-weighted images and hyperintensity with the spinal cord on T2-weighted images (8). Choi et al presented the MRI results of 3 patients and showed tumor hyperintensity on T2-weighted images and isointensity relative to the spinal cord on T1-weighted images (7). The MRI results of the present case were in agreement with these previous observations.

The differential diagnosis for spinal intradural capillary hemangioma includes a number of tumors, including neurinomas, meningiomas, hemangiopericytomas, hemangioendotheliomas, schwannnomas, cavernous angiomas, angiolipomas, solitary fibrous tumor paragangliomas, lymphomas, sarcoidosis, ependymomas and metastasis. MRI plays an important role in differentiating between these tumors. For example, meningiomas show isointensity or slight hypointensity on T1-weighted images, and isointensity or slight hyperintensity on T2-weighted images (7). On MRI, necrosis or cystic changes are frequently observed within neurimomas (7). A heterogeneous salt and pepper-like appearance of vascular signal voids may be present in paraganglioma on MRI (7). Therefore, the clinical utility of MRI should be fully accepted in the evaluation of intradural extramedullary masses.

A hemangioma may be classified as a capillary, cavernous or mixed hemangioma. It is possible to differentiate capillary hemangioma from other hemangioma by means of histological examination. The classic feature of capillary hemangiomas is a tumor composed of tightly packed capillary-sized vessels lined by a single layer of endothelial cells on microscopy (6). Therefore, the presence of different histological examination features is useful and effective in distinguishing capillary hemangioma from other tumors.

The treatment of choice for intradural extramedullary capillary hemangioma is complete surgical excision. In the present study, a laminectomy and durotomy were performed to access to the intradural space, and then a total tumor resection was performed. Due to the excessive vascularity of intradural extramedullary capillary hemangioma, a complete tumor resection should be performed, and a piece and piece resection should be avoided (6). We believe that, as bleeding of the intradural extramedullary capillary hemangioma may cause acute spinal cord compression, surgery should be performed immediately after an intradural extramedullary capillary hemangioma is found. Other treatments, including radiation therapy and embolization methods, have not been investigated in the therapy of intradural extramedullary capillary hemangioma in previously reported cases (6). Tumor recurrence has not been reported in previous studies, and no recurrence was found in the present case. Roncaroli et al (5) reported the cases of 2 patients who underwent complete resections of spinal capillary hemangiomas, with no recurrence 10 years after surgery. However, due to the potential for recurrence, long-term follow-up is required in such cases.

In conclusion, the current study presents an extremely rare case of spinal intradural capillary hemangioma in a 59-year-old woman. Surgical intervention was successfully performed and the patient's neurological symptoms improved. This case suggests that capillary hemangioma should be included in the differential diagnosis when an ovoid, well-demarcated mass is observed in the spinal intradural space, and that surgical treatment should be strongly considered in such cases.

\section{References}

1. Tokuda Y, Uozumi T, Sakoda K, Yamada K, Yamanaka M, Nomura S and Hamasaki T: Giant congenital capillary hemangioma of pericranium-case report. Neurol Med Chir (Tokyo) 30: 1029-1033, 1990.

2. Hida K, Tada M, Iwasaki Y and Abe H: Intramedullary disseminated capillary haemangioma with localized spinal cord swelling: Case report. Neurosurgery 33: 1099-1101, 1993.

3. Holtzman RN, Brisson PM, Pearl RE and Gruber ML: Lobular capillary hemangioma of the cauda equina. Case report. J Neurosurg 90 (2 Suppl): 239-241, 1999.

4. Roncaroli F, Scheitauer BW and Krauss WE: Hemangioma of spinal nerve root. J Neurosurg 91 (2 Suppl): 175-180, 1999.

5. Roncaroli F, Scheitauer BW and Krauss WE: Capillary hemangioma of the spinal cord. Report of four cases. J Neurosurg 93 (1 Suppl): 148-151, 2000.

6. Nowak DA and Widennka DC: Spinal intradural capillary haemangioma: A review. Eur Spine J 10: 464-472, 2001

7. Choi BY, Chang KH, Choe G, Han MH, Park SW, Yu IK, Park YH and Kim HJ: Spinal intradural extramedullary capillary hemangioma: MR imaging findings. AJNR Am J Neuroradiol 22: 799-802, 2001.

8. Shin JH, Lee HK, Jeon SR and Park SH: Spinal intradural capillary hemangioma: MR findings. AJNR Am J Neuroradiol 21: 954-956, 2000. 